Editor's Note: These short, critical reviews of recent papers in the Journal, written exclusively by graduate students or postdoctoral fellows, are intended to summarize the important findings of the paper and provide additional insight and commentary. For more information on the format and purpose of the Journal Club, please see http://www.jneurosci.org/misc/ifa_features.shtml.

\title{
Broadband Spectral Change: Evidence for a Macroscale Correlate of Population Firing Rate?
}

\author{
Kai J. Miller \\ Neurobiology and Behavior, University of Washington, Seattle, WA, 98195 \\ Review of Manning et al.
}

\section{Introduction}

In 1972, Brindley and Craggs measured the electric potential from the surface of the baboon brain using a 1-mm-diameter electrode. They found that the power in the $80-250 \mathrm{~Hz}$ frequency range of the electric potential time series was dynamically increased in motor areas during movement. Sites $2 \mathrm{~mm}$ apart from one another were specific for different movements of the same limb. This observation, that power in the high-frequency portion of the brain surface electric potential was specific for local cortical activity, was again demonstrated in electrocorticography (ECoG) by Crone et al. (1998) for different functions in distant regions of the human brain. Both groups proposed that this high-frequency power was a correlate of specific cortical activity, but it was unclear what this power increase meant at the neuronal level. More recently, Miller et al. (2009a,b) proposed and demonstrated that these observed high-frequency power changes actually reflected "broadband" power spectral change, across all frequencies. The low-frequency portion of these broadband changes was often obscured at

Received Dec. 27, 2009; revised Jan. 20, 2010; accepted Jan. 21, 2010

K.J.M. is supported by the National Aeronautics and Space Administration Graduate Student Research Program and the National Institutes of Health-National Institute of General Medical Sciences Medical Scientist Training Program. I thank Dora Hermes and Teresa Esch for reading of this manuscript.

Correspondence should be addressed to Kai J. Miller, Neurobiology and Behavior, University of Washington, Allen Building, Box 352350, Seattle, WA, 98195. E-mail: kjmiller@u.washington.edu.

DOI:10.1523/JNEUROSCI.6401-09.2010

Copyright $\odot 2010$ the authors $\quad 0270-6474 / 10 / 306477-03 \$ 15.00 / 0$ lower frequencies by coincident changes in rhythmic phenomena (e.g., $\alpha$ and $\beta$ ), so that only the high-frequency portion of the broadband change was observed. These broadband changes have a particular form (a power law in the frequency domain) (Fig. 1) and capture functionally specific cortical activity with a temporal precision of tens of milliseconds (Miller et al., 2009a,b). A recent Journal of Neuroscience article by Manning and colleagues (2009) directly shed light on the neurophysiologic nature of these broadband changes by measuring what aspects of the power spectral density (PSD) of the local field potential (LFP) correspond with single-neuron firing rates measured at the same cortical site.

Manning and colleagues (2009) performed the following experiment: in the course of treatment for epilepsy, penetrating microwires were transiently implanted in 20 human patients during the clinical identification of seizure foci. Each patient participated in a spatial navigation task while single-neuron action potential (AP) firing rates and the surrounding LFPs were measured from an array of microwires throughout different brain sites. The firing rate of each neuron and the corresponding normalized PSD of the LFP were calculated in half-second epochs. For each epoch, the power in the PSD was extracted in five discrete frequency ranges: delta (2-4 $\mathrm{Hz})$, theta $(4-8 \mathrm{~Hz})$, alpha $(8-12 \mathrm{~Hz})$, beta $(12-30 \mathrm{~Hz})$, and gamma $(30-150$ $\mathrm{Hz}$ ). In addition, an estimate of the broadband power in the PSD, across all frequencies, was obtained from each epoch. The firing rate was then compared with each power spectral feature using a regression approach, and an associated significance level was estimated by resampling (randomly time-shifting the LFP and AP event times with respect to one another to obtain a surrogate distribution). The best predictor of firing rate was the broadband feature of the PSD. There is a clear relation between increased firing rate and increased broadband power in the LFP [Manning et al. (2009), their Figs. 1 and 2]. This relation was robust, significant, and reproduced across a large number of individuals and brain sites. Manning and colleagues (2009) experimentally demonstrated, for the first time, that broadband spectral change in the electric potential is correlated with neuronal AP firing rate.

In the same week that the article by Manning et al. (2009) was published, Whittingstall and Logothetis (2009) published an article showing that $30-$ $100 \mathrm{~Hz}$ aspects of the LFP are significant predictors of multineuron firing rate; it is likely that this high-frequency change reflects a broadband change and represents a secondary confirmation of the finding by Manning et al. (2009). The electrical potential from both studies was measured at the spatial scale of the LFP, which has recently been demonstrated to reflect neuronal activity within $\sim 250 \mu \mathrm{m}$ of the recording electrode (Katzner et al., 2009). Because this 
broadband spectral change is correlated with the action potential rate at the LFP scale, broadband electric potential spectral changes may generically represent mean firing rate at larger scales as well. If true at larger scales, then the spatial scale that the recording electrode reflects would then dictate the size of the neuronal population that the firing rate is being averaged over. Seen in this light, the article by Manning et al. (2009) provides empirical evidence that broadband (or associated high-frequency) changes observed at larger spatial scales, in ECoG, are a correlate of the mean firing rate of the neuronal population beneath each recording electrode.

How might the reader gain intuition for the measured correlation in terms of neurophysiology? From a modeling perspective, heuristics for the relationship between changes in action potential rate and broadband, power-law, changes can be constructed relatively simply. Properties of the physiology underlying the current source density (CSD) in different cortical lamina were established experimentally in the late 1970s and early 1980s (Mitzdorf, 1985). Propagating action potentials in axons and axon terminals does not contribute strongly to the CSD at spatial scales of $\sim 50$ to $\geq 300 \mu \mathrm{m}$, e.g., the scales where CSD varies, LFPs pool from, or macroscale ECoG potentials average over. Instead, dendritic synaptic current influx and efflux modulate the CSD and, by extension, the LFP and the ECoG-scale potential. Emerging in vivo, simultaneous recordings of intracellular potential and LFP by Okun et al. (2009) show that the LFP and single-neuron transmembrane potential are tightly coupled temporally, independent of the spiking pattern of the neuron. This implies that the correlation observed by Manning et al. (2009) likely reflects the postsynaptic influence on many neighboring neurons by the neuron whose action potential times are being measured, and this may very well be augmented by redundant firing patterns across neighbors.

A very simple model for producing broadband spectral changes from changes in firing rate can be illustrated to provide intuition for this correlation, and also to illustrate why the experimental findings of Manning et al. (2009) provide evidence for a macroscale correlate in population firing rate. A model based on original research by Bédard et al. (2006) (later extended in Miller et al., 2009b) shows how the time course of the intracellular

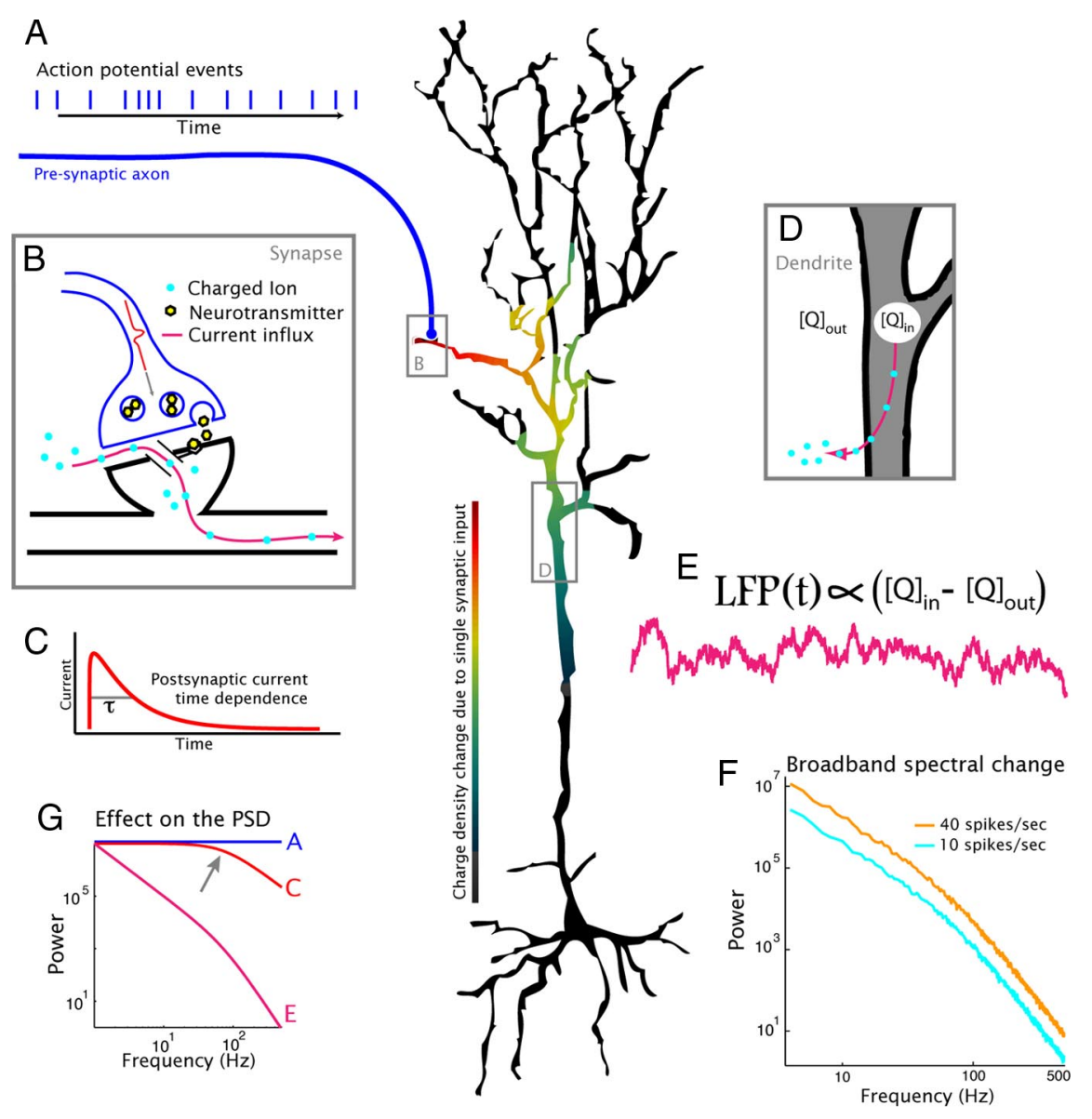

Figure 1. A heuristic model for how broadband spectral increases might emerge from increases in presynaptic AP firing rate. $A$, Poisson-distributed presynaptic APs arrive at a neighboring neuron. The PSD of these AP events over time has a flat, frequency-independent form (i.e., a "white noise" shape, with spikes coming with equal probability at every frequency; blue trace in $\mathbf{G})$. $\boldsymbol{B}$, At the synapse between the two neurons, each arriving AP triggers release of a neurotransmitter and postsynaptic current influx. As shown in the central schematic neuron, this results in a gradient of charge density within the dendritic arbor. $C$, The temporal shape of the postsynaptic current smears out the PSD, giving it a $1 / \mathrm{f}^{2}$ form, with a "kink" at a particular frequency determined by the decay time, $\tau$, of the postsynaptic current (here $\sim 70 \mathrm{~Hz}$ ), shown with a gray arrow in $\mathbf{G}$. D, In this model, the inputs from 6000 such synaptic currents are integrated over time and space, simulating the time-dependent change in transmembrane charge concentration. The associated transmembrane potential produces a time-dependent current across the dendritic membrane. $\boldsymbol{E}$, The combined effect of synaptic and transdendritic current influx/efflux induces a gradient of current-source density in the surrounding medium. The time dependence of transmembrane potentials are mimicked by the LFP (Okun et al., 2009), and likely by the macroscale (EC0G) potential as well. $\boldsymbol{F}$, The PSD shifts associated with changes in mean firing rate from presynaptic inputs are broadband in nature (spread across all frequencies), with a characteristic $P \sim 1 / f^{\chi}$ form (i.e., the power in the PSD falls off with increasing frequency according to the exponent, $\chi$ ). $\boldsymbol{G}$, The $P \sim 1 / f \chi$ PSD structure might emerge from the combinations of three simple processes. The first is Poisson-distributed input spikes [as in $\boldsymbol{A}$, reflected in the rate measured by Manning et al. (2009)]. The second is a characteristic postsynaptic current with exponential decay, which produces a $1 / \mathrm{f}^{2}$ form following a kink (gray arrow) at a frequency determined by the decay time at the synapse. The last process to shape the PSD is the integration of inward currents over time in the dendrite (as in $\boldsymbol{D}$ and $\boldsymbol{E}$ ). This model demonstrates how the relationship between firing rate and broadband change observed by Manning et al. (2009) might arise. Model adopted from Bédard et al. (2006) and Miller et al. (2009b).

dendritic charge concentration might result from spatiotemporal summation of postsynaptic current influxes from each arriving AP (Fig. 1). The broadband in the PSD results from the noise-like distribution of AP arrival times, and its $1 / \mathrm{f}$ falloff with frequency results from the shape of the synaptic current decay and the effect of temporal integration in the dendritic arbor. The Manning et al. (2009) finding supports models of this type, where basic phenomena, firing rate changes, produce spatially larger scale field potential changes. Furthermore, the strong correlation between firing rate and broadband spectral change in the electrical potential demonstrated empirically by Manning et al. (2009) provides powerful evidence that broadband power spectral changes observed at larger spatial scales may be a generic correlate of mean population firing rate. 


\section{References}

Bédard C, Kröger H, Destexhe A (2006) Does the $1 / \mathrm{f}$ frequency scaling of brain signals reflect self-organized critical states? Phys Rev Lett 97:118102.

Brindley GS, Craggs MD (1972) The electrical activity in the motor cortex that accompanies voluntary movement. J Physiol 223: 28P-29P.

Crone NE, Miglioretti DL, Gordon B, Lesser RP (1998) Functional mapping of human sensorimotor cortex with electrocorticographic spectral analysis. II. Event-related synchronization in the gamma band. Brain 121:2301-2315.
Katzner S, Nauhaus I, Benucci A, Bonin V, Ringach DL, Carandini M (2009) Local origin of field potentials in visual cortex. Neuron 61:35-41.

Manning JR, Jacobs J, Fried I, Kahana MJ (2009) Broadband shifts in LFP power spectra are correlated with single-neuron spiking in humans. J Neurosci 29:13613-13620.

Miller KJ, Zanos S, Fetz EE, den Nijs M, Ojemann JG (2009a) Decoupling the cortical power spectrum reveals real-time representation of individual finger movements in humans. J Neurosci 29:3132-3137.

Miller KJ, Sorensen LB, Ojemann JG, den Nijs M (2009b) Power-law scaling in the brain sur- face electric potential. PLoS Comput Biol 5:e1000609.

Mitzdorf U (1985) Current source-density method and application in cat cerebral cortex: investigation of evoked potentials and EEG phenomena. Physiol Rev 65:37-100.

Okun M, Naim A, Lampl I (2010) The subthreshold relation between cortical local field potential and neuronal firing unveiled by intracellular recordings in awake rats. J Neurosci 30:4440-4448.

Whittingstall K, Logothetis NK (2009) Frequencyband coupling in surface EEG reflects spiking activity in monkey visual cortex. Neuron 64:281-289. 